\title{
Deciding Simulations on Probabilistic Automata *
}

\author{
Lijun Zhang and Holger Hermanns
}

Department of Computer Science, Saarland University, Saarbrücken, Germany

\begin{abstract}
Probabilistic automata are a central model for concurrent systems exhibiting random phenomena. This paper presents, in a uniform setting, efficient decision algorithms for strong simulation on probabilistic automata, but with subtly different results. The algorithm for strong probabilistic simulation is shown to be of polynomial complexity via a reduction to LP problem, while the algorithm for strong simulation has complexity $\mathcal{O}\left(m^{2} n\right)$. The former relation allows for convex combinations of transitions in the definition and is thus less discriminative than the latter. As a byproduct, we obtain minimisation algorithms with respect to strong simulation equivalences and - for Markov decision processes - also to strong bisimulation equivalences. When extending these algorithms to the continuous-time setting, we retain same complexities for both strong and strong probabilistic simulations.
\end{abstract}

\section{Introduction}

Randomization has been employed widely for performance and dependability models, and consequently the study of verification techniques of probabilistic systems has drawn a lot of attention in recent years. In this paper, we consider probabilistic automata (PAs) in the style of Segala \& Lynch [12], which extend transition systems with probabilistic selection. They constitute a natural model of concurrent computation involving random phenomena. In a nutshell, a labelled transition in some PA leads to a probability distribution over the set of states, rather than a single state. The resulting model thus exhibits both nondeterministic choice (as in labelled transition systems) and probabilistic choice (as in Markov chains). A special case of PAs is formed by Markov decision processes (MDPs) which in their standard form do not have nondeterminism between the equally-labelled transitions [10].

Similar to the transition system setting, strong bisimulation and strong simulation relations $[9,8,12]$ have been proposed as means to compare the stepwise behaviour of states in PAs. Intuitively, state $s$ is simulated by another state $s^{\prime}$, formally $s \precsim s^{\prime}$ (" $s^{\prime}$ simulates $s$ "), if state $s^{\prime}$ can mimic all stepwise behaviours of $s$; the converse, i. e., $s^{\prime} \precsim s$ is not necessarily guaranteed, so state $s^{\prime}$ may perform steps that cannot be matched by $s$. In the non-probabilistic setting, $s \precsim s^{\prime}$

\footnotetext{
* This work is supported by the NWO-DFG bilateral project VOSS and by the DFG as part of the Transregional Collaborative Research Center SFB/TR 14 AVACS.
} 
requires every successor of $s$ via some action $\alpha$ to be related to a corresponding successor of $s^{\prime}$ reachable via the same action $\alpha$. For PAs, the above condition is lifted to distributions: It is required that every successor distribution of $s$ via action $\alpha$, called $\alpha$-successor distribution, has a corresponding $\alpha$-successor distribution at $s^{\prime}$. The correspondence of distributions is naturally defined with the concept of weight functions [8].

In the context of model checking, strong simulation relations can be used to combat the infamous state space explosion problem, owed to the preservation of PCTL-safety formulas [12]. The kernel of strong simulation, i. e., strong simulation equivalence, preserves both safe and live fragments of PCTL. Therefore, one can perform model checking on the quotient induced by these equivalences, if interested in safety or liveness properties. Since strong simulation equivalence is strictly coarser than strong bisimulation, the induced quotient automaton is also smaller.

All statements in the above paragraph stay perfectly valid if considering "strong probabilistic simulation" instead of "strong simulation". The former [12] is a relaxation of the latter in the sense that it enables convex combinations of multiple distributions belonging to equally labelled transitions. More concretely, assume that a state $s$ has no $\alpha$-successor distribution which can be related to an $\alpha$-successor distribution of $s^{\prime}$, yet there exists such a so-called $\alpha$-combined transition, a convex combination of several $\alpha$-successor distributions. Strong probabilistic simulation accounts for this and is thus coarser than strong simulation, but still preserves the same class of PCTL-properties as strong simulation does. Since it is coarser, the induced simulation quotient is potentially again smaller.

Cattani and Segala [6] have presented decision algorithms for strong (probabilistic) bisimulation for PAs. They reduced the decision problems to linear programming (LP) problems. In this paper, we will focus on decision algorithms for strong (probabilistic) simulation and strong (probabilistic) simulation equivalence for PAs. We will also extend the notion of strong (probabilistic) simulation to the continuous-time setting and study corresponding decision algorithms.

To compute the coarsest strong simulation for PAs, Baier et. al. [2] presented an algorithm which reduces the query whether a state strongly simulates another to a maximum flow problem. Their algorithm has complexity $\mathcal{O}\left(\left(m n^{6}+m^{2} n^{3}\right) / \log n\right)$ for $\mathrm{PAs}^{1}$, where $n$ denotes the number of states and $m$ denotes the number of transitions. For Markov chains, we have presented an algorithm [14] with complexity $\mathcal{O}\left(m^{2} n\right)$. This algorithm is also based on maximum flows, however it exploits the similarity of successive network flows across iterations. In the present paper, we extend that algorithm to the PA case and retain its complexity of $\mathcal{O}\left(m^{2} n\right)$. Especially in the very common case, where the state fanout of a model is bounded by a constant $k$ (and hence $m \leq k n$ ), our strong simulation algorithm has complexity $\mathcal{O}\left(n^{2}\right)$. The computational complexity of strong probabilistic simulation has not been tackled yet. We show that it can be determined by solving LP problems. As a byproduct of the decision algorithms

\footnotetext{
${ }^{1}$ Note that the $m$ used here is slight different from the $m$ as we use it. A detailed comparison is provided later, in Remark 1 of Section 4.
} 
for strong simulation preorders, we obtain one for strong simulation equivalences $\precsim \cap \precsim^{-1}$. For the special case of MDPs [10], which arise from PAs by disallowing nondeterministic choices of equally labelled transitions and sub-stochastic distributions, strong simulation equivalence and strong bisimulation coincide [1]. We thus obtain a decision algorithm for computing strong bisimulations for MDPs.

Further, we consider continuous-time probabilistic automata (CPAs), the continuous-time counterpart of PAs. We give decision algorithms for strong simulation and strong probabilistic simulation on CPAs. For both of them, we show that the decision algorithm have the same complexity as the corresponding one for PAs.

In summary, our paper makes the following contributions: It presents novel decision algorithms for strong (probabilistic) simulation on discrete-time and continuous-time probabilistic automata (PAs, CPAs), and does so in a uniform way. As special cases, discrete-time and continuous-time Markov decision processes are considered, where in particular, our results yield an efficient algorithm to compute strong bisimulations.

Organisation of this paper. In Section 2 we recall necessary definitions of models and relations we consider. Section 3 recalls how to effectively compute strong simulation relations on fully probabilistic systems. Then, we extend the algorithm in Section 4 to deal with PAs, and show that strong probabilistic simulation can be computed by solving LP problems. We will also discuss strong (probabilistic) simulation equivalences. The algorithms are extended to CPAs in Section 5. Section 6 concludes the paper.

\section{Preliminaries}

This section introduces the basic models and simulation relations we consider.

Models. Let $A P$ be a fixed, finite set of atomic propositions. Let $X, Y$ be finite sets. For $f: X \rightarrow \mathbb{R}$, let $f(A)$ denote $\sum_{x \in A} f(x)$ for all $A \subseteq X$. If $f: X \times Y \rightarrow \mathbb{R}$ is a two-dimensional function, let $f(x, A)$ denote $\sum_{y \in A} f(x, y)$ for all $x \in X$ and $A \subseteq Y$, and $f(A, y)$ denote $\sum_{x \in A} f(x, y)$ for all $y \in Y$ and $A \subseteq X$. For a finite set $S$, a distribution $\mu$ on $S$ is a function $\mu: S \rightarrow[0,1]$ satisfying the condition $\mu(S) \leq 1$. The support of $\mu$ is defined by $\operatorname{Supp}(\mu)=\{s \mid \mu(s)>0\}$, and the size of $\mu$ is defined by $|\mu|=|\operatorname{Supp}(\mu)|$. The distribution $\mu$ is called stochastic if $\mu(S)=1$, absorbing if $\mu(S)=0$, and sub-stochastic otherwise. We use an auxiliary state (not a real state) $\perp \notin S$ and set $\mu(\perp)=1-\mu(S)$. Note that $\mu(\perp)>0$ if $\mu$ is not stochastic. Further, let $S_{\perp}$ denote the set $S \cup\{\perp\}$, and let $S_{u p p}(\mu)=$ $\operatorname{Supp}(\mu) \cup\{\perp\}$ if $\mu(\perp)>0$. We let $\operatorname{Dist}(S)$ denote the set of distributions over the set $S$. We recall the definition of probabilistic automata [12]:

Definition 1. A probabilistic automaton (PA) is a tuple $\mathcal{M}=(S$, Act $, \mathbf{P}, L)$ where $S$ is a finite set of states, Act is a finite set of actions, $\mathbf{P} \subseteq S \times$ Act $\times$ $\operatorname{Dist}(S)$ is a finite set, called the probabilistic transition matrix, and $\bar{L}: S \rightarrow 2^{A P}$ is a labeling function. 
For $(s, \alpha, \mu) \in \mathbf{P}$, we use $s \stackrel{\alpha}{\rightarrow} \mu$ as a shorthand notation, and call $\mu$ an $\alpha$-successor distribution of $s$. Let $\operatorname{Act}(s)=\{\alpha \mid \exists \mu: s \stackrel{\alpha}{\rightarrow} \mu\}$ denote the set of actions enabled at $s$. For $s \in S$ and $\alpha \in \operatorname{Act}(s)$, let $\operatorname{Steps}_{\alpha}(s)=\{\mu \in \operatorname{Dist}(S) \mid$ $s \stackrel{\alpha}{\rightarrow} \mu\}$ and $\operatorname{Steps}(s)=\bigcup_{\alpha \in \operatorname{Act}(s)} \operatorname{Steps}_{\alpha}(s)$. We introduce the notion of fanout for $\mathcal{M}$. The fanout of a state $s$ is defined by fan $(s)=\sum_{\alpha \in \operatorname{Act}(s)} \sum_{\mu \in \text { Steps }_{\alpha}(s)}|\mu|$. Intuitively, $\operatorname{fan}(s)$ denote the total sum of size of outgoing distributions of state $s$. The fanout of $\mathcal{M}$ is defined by $\max _{s \in S} \operatorname{fan}(s)$.

A Markov decision process (MDP) [10] arises from the PA $\mathcal{M}$ such that for $s \in S$ and $\alpha \in A c t$, there is at most one $\alpha$-successor distribution $\mu$ of $s$ which must be stochastic. $\mathcal{M}$ is a fully probabilistic system (FPS) if for $s \in S$, there is at most one transition $s \stackrel{\alpha}{\rightarrow} \mu$. A discrete-time Markov chain (DTMC) is a FPS where all distributions are either stochastic or absorbing. For ease of notation, we give a simpler definition for FPSs by dropping the set of actions:

Definition 2. An FPS is a tuple $\mathcal{D}=(S, \mathbf{P}, L)$ where $S, L$ as defined for PAs, and $\mathbf{P}: S \times S \rightarrow[0,1]$ is the probabilistic transition matrix such that $\mathbf{P}(s, \cdot) \in$ $\operatorname{Dist}(S)$ for all $s \in S$.

Strong simulation relations. Strong simulation requires that every $\alpha$-successor distribution of one state have a corresponding $\alpha$-successor distribution of the other state. The correspondence of distributions is naturally defined with the concept of weight functions [8].

Definition 3. Let $\mu, \mu^{\prime} \in \operatorname{Dist}(S)$ and $R \subseteq S \times S$. A weight function for $\left(\mu, \mu^{\prime}\right)$ with respect to $R$, denoted by $\mu \sqsubseteq_{R} \mu^{\prime}$, is a function $\Delta: S_{\perp} \times S_{\perp} \rightarrow[0,1]$ such that $\Delta\left(s, s^{\prime}\right)>0$ implies $s R s^{\prime}$ or $s=\perp, \mu(s)=\Delta\left(s, S_{\perp}\right)$ for $s \in S_{\perp}$ and $\mu^{\prime}\left(s^{\prime}\right)=\Delta\left(S_{\perp}, s^{\prime}\right)$ for $s^{\prime} \in S_{\perp}$.

Now we recall the definition of strong simulation for PAs $[12,8]$ :

Definition 4. Let $\mathcal{M}=(S$, Act, $\mathbf{P}, L)$ be a $P A . R \subseteq S \times S$ is a strong simulation on $\mathcal{M}$ iff for all $s_{1}, s_{2}$ with $s_{1} R s_{2}: L\left(s_{1}\right)=L\left(s_{2}\right)$ and if $s_{1} \stackrel{\alpha}{\rightarrow} \mu_{1}$ then there exists a transition $s_{2} \stackrel{\alpha}{\rightarrow} \mu_{2}$ with $\mu_{1} \sqsubseteq_{R} \mu_{2}$. We write $s_{1} \precsim \mathcal{M} s_{2}$ iff there exists a strong simulation $R$ on $\mathcal{M}$ such that $s_{1} R s_{2}$.

We say also that $s_{2}$ strongly simulates $s_{1}$ in $\mathcal{M}$ iff $s_{1} \precsim \mathcal{M} s_{2}$. Obviously $\precsim \mathcal{M}$ is the coarsest strong simulation relation for $\mathcal{M}$.

Simulation up to $R$. For an arbitrary relation $R$ on the state space $S$ of $\mathcal{M}$ with $s_{1} R s_{2}$, we say that $s_{2}$ simulates $s_{1}$ strongly up to $R$, denoted $s_{1} \precsim R s_{2}$, if $L\left(s_{1}\right)=L\left(s_{2}\right)$ and if $s_{1} \stackrel{\alpha}{\rightarrow} \mu_{1}$ then there exists a transition $s_{2} \stackrel{\alpha}{\rightarrow} \mu_{2}$ with $\mu_{1} \sqsubseteq_{R} \mu_{2}$. Otherwise we write $s_{1} \mathscr{L}_{R} s_{2}$. Note that $s_{1} \precsim_{R} s_{2}$ does not imply $s_{1} \precsim \mathcal{M} s_{2}$ unless $R$ is a strong simulation, since only the first step is considered for $\precsim R$.

Strong Probabilistic Simulation Relations. We recall first the notion of combined transition [12], a convex combination of several equally labelled transitions: 
Definition 5. Let $\mathcal{M}=(S$, Act $, \mathbf{P}, L)$ be a PA. Assume that Steps ${ }_{\alpha}(s)=$ $\left\{\mu_{1}, \ldots, \mu_{k}\right\}$ where $k=\mid$ Steps $_{\alpha}(s) \mid$. The tuple $(s, \alpha, \mu)$ is a combined transition, denoted by $s \stackrel{\alpha}{\rightarrow} C \mu$, iff there exist constants $c_{1}, \ldots, c_{k} \in[0,1]$ with $\sum_{i=1}^{k} c_{i}=1$ such that $\mu(s)=\sum_{i=1}^{k} c_{i} \mu_{i}(s)$ for all $s \in S$.

Strong probabilistic simulation is insensitive to combined transitions [12], thus, it is a relaxation of strong simulation. It is coarser than strong simulation, but still preserves the same class of PCTL-properties as strong simulation does. The key difference to Definition 4 is the use of $\stackrel{\alpha}{\rightarrow}_{C}$ instead of $\stackrel{\alpha}{\rightarrow}$ :

Definition 6. Let $\mathcal{M}=(S$, Act $, \mathbf{P}, L)$ be a $P A . R \subseteq S \times S$ is a strong probabilistic simulation on $\mathcal{M}$ iff for all $s_{1}, s_{2}$ with $s_{1} R s_{2}: L\left(s_{1}\right)=L\left(s_{2}\right)$ and if $s_{1} \stackrel{\alpha}{\rightarrow} \mu_{1}$ then there exists a combined transition $s_{2} \stackrel{\alpha}{\rightarrow} C \mu_{2}$ with $\mu_{1} \sqsubseteq_{R} \mu_{2}$. We write $s_{1} \precsim_{\mathcal{M}}^{p} s_{2}$ iff there exists a strong probabilistic simulation $R$ on $\mathcal{M}$ such that $s_{1} R s_{2}$.

Similar to strong simulation, $\precsim_{\mathcal{M}}^{p}$ is the coarsest strong probabilistic simulation relation for $\mathcal{M}$. The definition of simulation up to $R$ for strong simulation $\left(\precsim_{R}\right)$ carries over directly to strong probabilistic simulation, denoted by $\left(\precsim_{R}^{p}\right)$. Since MDPs can be considered as special PAs, we obtain the notion of strong simulation and strong probabilistic simulation for MDPs. Moreover, strong simulation and strong probabilistic simulation trivially coincide for MDPs as, by definition, for each state there is at most one successor distribution per action. Note that the above statements are also true for FPSs.

\section{Algorithms for Fully Probabilistic Systems}

In this section, we briefly review the algorithm to decide strong simulation preorder for FPSs. For more detail, we refer to [14], where the maximum flow problem and the preflow algorithm for computing maximum flow are also repeated, which are key components of the decision algorithms to be presented. As DTMCs are special FPSs, the algorithm applies directly.

Firstly, we discuss the decisive part of the algorithm: The check whether $s_{2}$ strongly simulates $s_{1}$ up to a relation $R$, i. e., $s_{1} \precsim_{R} s_{2}$. As the condition $L\left(s_{1}\right)=$ $L\left(s_{2}\right)$ is easy to check, we need to check whether $\mathbf{P}\left(s_{1}, \cdot\right) \sqsubseteq_{R} \mathbf{P}\left(s_{2}, \cdot\right)$ holds. This is reduced to a maximum flow computation on the network $\mathcal{N}\left(\mathbf{P}\left(s_{1}, \cdot\right)\right.$, $\left.\mathbf{P}\left(s_{2}, \cdot\right), R\right)$ constructed out of $\mathbf{P}\left(s_{1}, \cdot\right), \mathbf{P}\left(s_{2}, \cdot\right)$ and $R$. This network is constructed via a graph containing a copy $\bar{t} \in \overline{S_{\perp}}$ of each state $t \in S_{\perp}$ where $\overline{S_{\perp}}=\left\{\bar{t} \mid t \in S_{\perp}\right\}$ defined as follows: Let $\uparrow$ (the source) and $\downarrow$ (the sink) be two additional vertices not contained in $S_{\perp} \cup \overline{S_{\perp}}$. For functions $\mu, \mu^{\prime}: S \rightarrow \mathbb{R}_{\geq 0}$ and a relation $R \subseteq S \times S$ we define the network $\mathcal{N}\left(\mu, \mu^{\prime}, R\right)=(V, E, u)$ with the set of vertices $V=\{\uparrow, \downarrow\} \cup \operatorname{Supp}_{\perp}(\mu) \cup \overline{\operatorname{Supp}_{\perp}\left(\mu^{\prime}\right)}$ and the set of edges $E$ defined by $E=\{(s, \bar{t}) \mid(s, t) \in R \vee s=\perp\} \cup\{(\uparrow, s),(\bar{t}, \downarrow)\}$ where $s \in \operatorname{Supp}_{\perp}(\mu)$ and $t \in S u p p_{\perp}\left(\mu^{\prime}\right)$. The capacity function $u$ is defined as follows: $u(\uparrow, s)=\mu(s)$ for all $s \in S_{\perp}, u(\bar{t}, \downarrow)=\mu^{\prime}(t)$ for all $t \in S_{\perp}, u(s, \bar{t})=\infty$ for all $(s, t) \in E$ and $u(v, w)=0$ otherwise. This network is a bipartite network, where the vertices can 
be partitioned into two subsets $V_{1}:=\operatorname{Supp}_{\perp}(\mu) \cup\{\downarrow\}$ and $V_{2}:=\overline{\operatorname{Supp}_{\perp}\left(\mu^{\prime}\right)} \cup\{\uparrow\}$ such that all edges have one endpoint in $V_{1}$ and another in $V_{2}$. For two states $s_{1}, s_{2}$ of an FPS, we let $\mathcal{N}\left(s_{1}, s_{2}, R\right)$ denote the network $\mathcal{N}\left(\mathbf{P}\left(s_{1}, \cdot\right), \mathbf{P}\left(s_{2}, \cdot\right), R\right)$. The following lemma [2] expresses the crucial relationship between maximum flows and weight functions:

Lemma 1. Let $S$ be a finite set of states and $R$ be a relation on $S$. Let $\mu, \mu^{\prime} \in$ $\operatorname{Dist}(S)$. Then, $\mu \sqsubseteq_{R} \mu^{\prime}$ iff the maximum flow in $\mathcal{N}\left(\mu, \mu^{\prime}, R\right)$ is 1 .

Thus we can decide $s_{1} \precsim_{R} s_{2}$ by computing the maximum flow in $\mathcal{N}\left(s_{1}, s_{2}, R\right)$. A key observation we made in [14] is that the networks $\mathcal{N}\left(s_{1}, s_{2}, \cdot\right)$ constructed later in successive iterations are very similar: They differ from iteration to iteration only by deletion of some edges induced by the successive clean up of $R$. The algorithm, which we shall repeat later, exploits this fact by leveraging preflow rather than re-starting maximum flow computation from scratch each time. Formally, we look at the network $\mathcal{N}\left(s_{1}, s_{2}, R_{\text {init }}\right)$ where $R_{\text {init }}=\left\{\left(s_{1}, s_{2}\right) \in\right.$ $\left.S \times S \mid L\left(s_{1}\right)=L\left(s_{2}\right)\right\}$. Let $D_{1}, \ldots, D_{k}$ be pairwise disjoint subsets of $R_{\text {init }}$, which correspond to the pairs deleted from $R_{\text {init }}$ in iteration $i$. Let $\mathcal{N}\left(s_{1}, s_{2}, R_{i}\right)$ denote $\mathcal{N}\left(s_{1}, s_{2}, R_{\text {init }}\right)$ if $i=1$, and $\mathcal{N}\left(s_{1}, s_{2}, R_{i-1} \backslash D_{i-1}\right)$ if $1<i \leq k+1$. Let $f_{i}$ denote the maximum flow of the network $\mathcal{N}\left(s_{1}, s_{2}, R_{i}\right)$ for $i=1, \ldots, k+1$. We address the problem of checking $\left|f_{i}\right|=1$ for all $i=1, \ldots, k+1$. Very similar to the parametric maximum algorithm [7, p. 34], the algorithm $\operatorname{SMF}_{\left(s_{1}, s_{2}\right)}$ (sequence of maximum flows) for the pair $\left(s_{1}, s_{2}\right)$ consists of initialising the preflow $f_{\left(s_{1}, s_{2}\right)}$ and the distance function $d_{\left(s_{1}, s_{2}\right)}$ as for the preflow algorithm, setting $i=0$, and repeating the following steps at most $k$ times:

$\operatorname{SMF}_{\left(s_{1}, s_{2}\right)}$

1. Increase $i$ by 1 . If $i=1$ go to step 2 . Otherwise, for all pairs $\left(u_{1}, u_{2}\right) \in D_{i-1}$, set $f_{\left(s_{1}, s_{2}\right)}\left(u_{1}, \overline{u_{2}}\right)=0$ and replace the flow $f_{\left(s_{1}, s_{2}\right)}\left(\overline{u_{2}}, \downarrow\right)$ by $f_{\left(s_{1}, s_{2}\right)}\left(\overline{u_{2}}, \downarrow\right)$ $-f_{\left(s_{1}, s_{2}\right)}\left(u_{1}, \overline{u_{2}}\right)$. Set $\mathcal{N}\left(s_{1}, s_{2}, R_{i}\right)=\mathcal{N}\left(s_{1}, s_{2}, R_{i-1} \backslash D_{i-1}\right)$. Let $f_{\left(s_{1}, s_{2}\right)}$ and $d_{\left(s_{1}, s_{2}\right)}$ be the resulting flow and final valid distance function.

2. Apply the preflow algorithm to calculate the maximum flow for $\mathcal{N}\left(s_{1}, s_{2}, R_{i}\right)$ with preflow $f_{\left(s_{1}, s_{2}\right)}$ and distance function $d_{\left(s_{1}, s_{2}\right)}$.

3. If $\left|f_{\left(s_{1}, s_{2}\right)}\right|<1$ return false for all $j \geq i$. Otherwise, return true and continue with step 1 .

To understand this algorithm, assume $i>1$. At step (1.), before we remove the edges $D_{i-1}$ from the network $\mathcal{N}\left(s_{1}, s_{2}, R_{i-1}\right)$, we modify the flow $f_{\left(s_{1}, s_{2}\right)}$, which is the maximum flow of the network $\mathcal{N}\left(s_{1}, s_{2}, R_{i-1}\right)$, by

- setting $f_{\left(s_{1}, s_{2}\right)}\left(u_{1}, \overline{u_{2}}\right)=0$ for all deleted edges $\left(u_{1}, u_{2}\right) \in D_{i-1}$, and

- modifying $f_{\left(s_{1}, s_{2}\right)}\left(\overline{u_{2}}, \downarrow\right)$ such that the flow $f_{\left(s_{1}, s_{2}\right)}$ becomes consistent with the flow conservation rule.

The excess $e(v)$ is increased if there exists $(v, w) \in D_{i-1}$ such that $f_{\left(s_{1}, s_{2}\right)}(v, w)>$ 0 , and unchanged otherwise. Hence, the modified flow is a preflow. The distance function $d_{\left(s_{1}, s_{2}\right)}$ keeps valid, since by removing the set of edges $D_{i-1}$, no new 


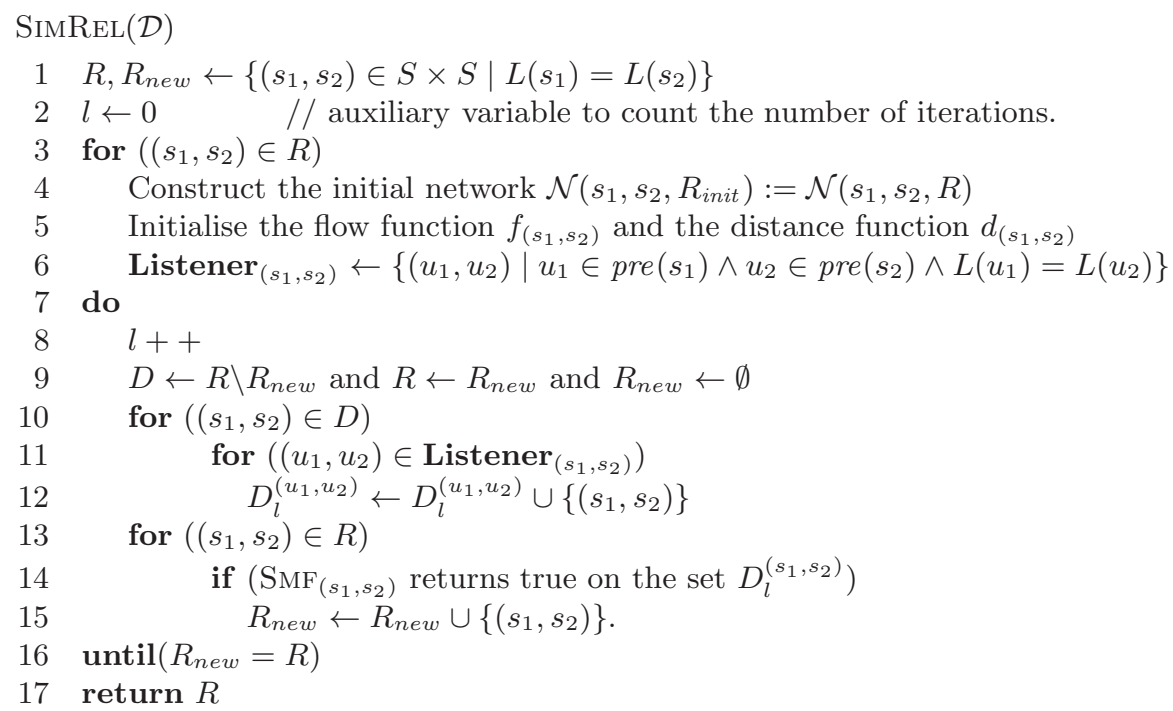

Fig. 1. Efficient algorithm for deciding strong simulation for FPSs

residual edges are induced. This guarantees that, at step (2.), the preflow algorithm finds a maximum flow over the network $\mathcal{N}\left(s_{1}, s_{2}, R_{i}\right)$. If $\left|f_{\left(s_{1}, s_{2}\right)}\right|<1$ at some iteration $i$, then $\left|f_{\left(s_{1}, s_{2}\right)}\right|<1$ for all iterations $j \geq i$ because more edges will be deleted in subsequent iterations. Therefore, at step (3.), the algorithm returns true and continues with step (1.) if $\left|f_{\left(s_{1}, s_{2}\right)}\right|=1$, otherwise, returns false for all subsequent iterations. Let $\operatorname{post}(s)$ denote $\operatorname{Supp}(\mathbf{P}(s, \cdot))$, i. e., the set of successor states of $s$. The complexity of the algorithm [14] is given by:

Lemma 2. Let $D_{1}, \ldots, D_{k}$ be pairwise disjoint subsets of $R_{\text {init }} \cap \operatorname{post}\left(s_{1}\right) \times$ $\operatorname{post}\left(s_{2}\right)$. Let $f_{i}$ denote the flow constructed at the end of step (2.) in iteration $i$. Assume that $\mid$ post $\left(s_{1}\right)|\leq|$ post $\left(s_{2}\right) \mid$. The algorithm $\operatorname{SMF}_{\left(s_{1}, s_{2}\right)}$ correctly computes maximum flow $f_{i}$ for $\mathcal{N}\left(s_{1}, s_{2}, R_{i}\right)$ where $i=1, \ldots, k+1$, and runs in time $\mathcal{O}\left(\left|\operatorname{post}\left(s_{1}\right)\right|\left|\operatorname{post}\left(s_{2}\right)\right|^{2}\right)$.

The algorithm SIMREL for deciding strong simulation for FPSs is depicted in Fig. 1. It takes the model $\mathcal{D}$ as a parameter. To calculate the strong simulation relation for $\mathcal{D}$, the algorithm starts with the trivial relation $R_{\text {init }}=\left\{\left(s_{1}, s_{2}\right) \in\right.$ $\left.S \times S \mid L\left(s_{1}\right)=L\left(s_{2}\right)\right\}$ (line 1 ). The variable $l$ (line 2 ) denotes the number of iterations of the until-loop, and the set $D$ (line 9 ) contains edges removed from $R$. For every pair $\left(s_{1}, s_{2}\right) \in R_{\text {init }}$, the network $\mathcal{N}\left(s_{1}, s_{2}, R_{\text {init }}\right)$ (line 4$)$, the flow function $f_{\left(s_{1}, s_{2}\right)}$ and the distance function $d_{\left(s_{1}, s_{2}\right)}$ are initialised as for the preflow algorithm (line 5). At line 6 a set

$\operatorname{Listener}_{\left(s_{1}, s_{2}\right)}=\left\{\left(u_{1}, u_{2}\right) \mid u_{1} \in \operatorname{pre}\left(s_{1}\right) \wedge u_{2} \in \operatorname{pre}\left(s_{2}\right) \wedge L\left(u_{1}\right)=L\left(u_{2}\right)\right\}$

is saved, where pre $(s)=\{t \in S \mid \mathbf{P}(t, s)>0\}$ is the set of predecessors of $s$. The set $\operatorname{Listener}_{\left(s_{1}, s_{2}\right)}$ contains all pairs $\left(u_{1}, u_{2}\right)$ such that the network $\mathcal{N}\left(u_{1}, u_{2}, R\right)$ 
contains the edge $\left(s_{1}, \overline{s_{2}}\right)$. In lines $10-12$, the pair $\left(s_{1}, s_{2}\right)$ is inserted into the set $D_{l}^{\left(u_{1}, u_{2}\right)}$ if $\left(s_{1}, s_{2}\right) \in D$ and $\left(u_{1}, u_{2}\right) \in \operatorname{Listener}_{\left(s_{1}, s_{2}\right)} \cdot D_{l}^{\left(u_{1}, u_{2}\right)}$ contains edges which should be removed to update the network for $\left(u_{1}, u_{2}\right)$ in iteration l. At line 14, the algorithm $\operatorname{SMF}_{\left(s_{1}, s_{2}\right)}$ constructs the maximum flow for the set $D_{l}^{\left(s_{1}, s_{2}\right)}$. Note that $l$ corresponds to $i$ in SMF. The initialisation of SMF corresponds to lines $4-5$. In the first iteration (in which $\left.D_{1}^{\left(s_{1}, s_{2}\right)}=\emptyset\right), \operatorname{SMF}_{\left(s_{1}, s_{2}\right)}$ skips the computations in step (1.) and proceeds directly to step (2.), in which the maximum flow $f_{1}$ for $\mathcal{N}\left(s_{1}, s_{2}, R_{\text {init }}\right)$ is constructed. In iteration $l>1, \operatorname{SMF}_{\left(s_{1}, s_{2}\right)}$ takes the set $D_{l}^{\left(s_{1}, s_{2}\right)}$, updates the flow $f_{l-1}$ and the network, and constructs the maximum flow $f_{l}$ for the network $\mathcal{N}\left(s_{1}, s_{2}, R_{l}\right)$. If $\operatorname{SMF}_{\left(s_{1}, s_{2}\right)}$ returns true, $\left(s_{1}, s_{2}\right)$ is inserted into $R_{\text {new }}$ and survives this iteration. Otherwise, $s_{2}$ cannot strongly simulate $s_{1}$ up to the current relation $R$, hence the pair $\left(s_{1}, s_{2}\right)$ is removed. This proceeds until there is no such pair left (line 16), i. e., $R_{\text {new }}=R$. Invariantly throughout the loop it holds that $R$ is coarser than $\precsim_{\mathcal{D}}$. Hence, we obtain the strong simulation preorder $\precsim_{\mathcal{D}}=R$, once the algorithm terminates (line 17). For a given FPS, let $m, n$ denote the number of transitions and states. Note that the fanout for FPSs is given by $\max _{s \in S}|\mathbf{P}(s, \cdot)|$. We recall the decisive complexity result from [14]:

Lemma 3. $\operatorname{SimReL}(\mathcal{D})$ runs in time $\mathcal{O}\left(m^{2} n\right)$ and in space $\mathcal{O}\left(m^{2}\right)$. If the fanout is bounded by a constant, it has complexity $\mathcal{O}\left(n^{2}\right)$, both in time and space.

\section{Algorithms for Probabilistic Automata}

In this section we present algorithms for deciding strong (probabilistic) simulations for PAs. First, we extend the algorithm SimREL to deal with strong simulation for PAs. For strong probabilistic simulation, we show that the algorithm can be reduced to LP problems. Finally, we discuss the strong (probabilistic) simulation equivalence for PAs.

Strong Simulations. We aim to extend SimREL in Fig. 1 to determine the strong simulation on PAs instead of FPSs. Assume that $L\left(s_{1}\right)=L\left(s_{2}\right)$. We consider line 14, which checks the condition $\mathbf{P}\left(s_{1}, \cdot\right) \sqsubseteq_{R} \mathbf{P}\left(s_{2}, \cdot\right)$ using SmF. By Definition 4 of strong simulation for PAs, we should check the condition

$$
\forall s_{1} \stackrel{\alpha}{\rightarrow} \mu_{1} . \exists s_{2} \stackrel{\alpha}{\rightarrow} \mu_{2} \text { with } \mu_{1} \sqsubseteq_{R} \mu_{2}
$$

instead. Recall the condition $\mu_{1} \sqsubseteq_{R} \mu_{2}$ is true iff the maximum flow of the network $\mathcal{N}\left(\mu_{1}, \mu_{2}, R\right)$ has value one. For notational convenience, this network is denoted by $\mathcal{N}\left(s_{1}, \alpha, \mu_{1}, s_{2}, \mu_{2}, R\right)$. We say that the check $\mu_{1} \sqsubseteq R \mu_{2}$ is successful if the corresponding maximum flow has value one, and unsuccessful otherwise.

Our goal is to carry out a sequence of checks on similar networks (obtained by successive clean up of $R$ ) using only a single call to a slightly adaption of the algorithm SMF. For any states $s_{1}, s_{2}$, action $\alpha$, consider the two transitions $s_{1} \stackrel{\alpha}{\rightarrow} \mu_{1}$ and $s_{2} \stackrel{\alpha}{\rightarrow} \mu_{2}$. Let $k\left(\mu_{1}, \mu_{2}\right)$ denote the number of successful checks of 
$\mu_{1} \sqsubseteq_{R} \mu_{2}$. We modify the algorithm SMF slightly such that the $k\left(\mu_{1}, \mu_{2}\right)$ successful checks for $\left(s_{1}, s_{2}\right)$, plus at most one unsuccessful check, can be performed by only a single call to SMF. To enable that, we take $\alpha, \mu_{1}, \mu_{2}$ as additional parameters for SMF. Now, for $\left(s_{1}, s_{2}\right) \in R$ with $s_{1} \stackrel{\alpha}{\rightarrow} \mu_{1}$ and $s_{2} \stackrel{\alpha}{\rightarrow} \mu_{2}$, the network $\mathcal{N}\left(s_{1}, \alpha, \mu_{1}, s_{2}, \mu_{2}, R\right)$ is constructed instead of $\mathcal{N}\left(s_{1}, s_{2}, R\right)$. Other parts of SMF remain unchanged. Denote the modified version by $\operatorname{SMF}^{\prime}\left(s_{1}, \alpha, \mu_{1}, s_{2}, \mu_{2}\right)$. Let $R_{\text {init }}=\left\{\left(s_{1}, s_{2}\right) \in S \times S \mid L\left(s_{1}\right)=L\left(s_{2}\right)\right\}$. As complexity of SMF' we get:

Lemma 4. Let $\left(s_{1}, s_{2}\right) \in R_{\text {init }}$. Consider the two transitions $s_{1} \stackrel{\alpha}{\rightarrow} \mu_{1}$ and $s_{2} \stackrel{\alpha}{\rightarrow}$ $\mu_{2}$. Let $D_{1}, \ldots, D_{k}$ be pairwise disjoint subsets of $R_{\text {init }} \cap \operatorname{Supp}\left(\mu_{1}\right) \times \operatorname{Supp}\left(\mu_{2}\right)$. Let $f_{i}$ denote the flow constructed at the end of step (2.) in iteration $i$ of SMF'. Assume that $\left|\mu_{1}\right| \leq\left|\mu_{2}\right|$. The algorithm $\mathrm{SMF}_{\left(s_{1}, \alpha, \mu_{1}, s_{2}, \mu_{2}\right)}$ correctly computes maximum flow $f_{i}$ for $\mathcal{N}\left(s_{1}, \alpha, \mu_{1}, s_{2}, \mu_{2}, R_{i}\right)$ where $i=1, \ldots, k+1$, and runs in time $\mathcal{O}\left(\left|\mu_{1}\right|\left|\mu_{2}\right|^{2}\right)$.

The algorithm SIMREL for deciding strong simulation for PAs is presented in Fig. 2. We use similar notations as Baier et. al. in [2]. During the initialisation (lines 2-8), for $\left(s_{1}, s_{2}\right) \in R$ and $s_{1} \stackrel{\alpha}{\rightarrow} \mu_{1}$, the set $\operatorname{Sim}_{\left(s_{1}, \alpha, \mu_{1}\right)}\left(s_{2}\right)$ is initialised to $\operatorname{Steps}_{\alpha}\left(s_{2}\right)$ (line 4). Intuitively, $\operatorname{Sim}_{\left(s_{1}, \alpha, \mu_{1}\right)}\left(s_{2}\right)$ contains all potential candidates of $\alpha$-successor distributions of $s_{2}$ which could be used to establish the condition $\mu_{1} \sqsubseteq_{R} \mu_{2}$ for the relation $R$ considered. The set $\operatorname{Sim}_{\left(s_{1}, \alpha, \mu_{1}\right)}\left(s_{2}\right)$ is represented as a list. We use the operation head $(\cdot)$ to get the first element of the list, and use the operation $\operatorname{tail}(\cdot)$ to remove the first element of the list. The operation empty $(\cdot)$ checks whether the list is empty. At line 5 , the first element of $\operatorname{Sim}_{\left(s_{1}, \alpha, \mu_{1}\right)}\left(s_{2}\right)$ is assigned to $\mu_{2}$. The network $\mathcal{N}\left(s_{1}, \alpha, \mu_{1}, s_{2}, \mu_{2}, R_{\text {init }}\right)$, preflow and distance function for it are initialised (lines 6-7) as for $\operatorname{SimReL}(\mathcal{D})$. Similarly, the set $\operatorname{Listener}_{\left(s_{1}, s_{2}\right)}$ for $\left(s_{1}, s_{2}\right)$ is introduced which contains tuples $\left(u_{1}, \alpha, \mu_{1}, u_{2}, \mu_{2}\right)$ such that the network $\mathcal{N}\left(u_{1}, \alpha, \mu_{1}, u_{2}, \mu_{2}, R_{\text {init }}\right)$ contains the edge $\left(s_{1}, \overline{s_{2}}\right)$.

In the main iteration, the sets $D, R, R_{\text {new }}$ and $D_{l}^{\left(u_{1}, \alpha, \mu_{1}, u_{2}, \mu_{2}\right)}$ are updated (lines 10-13) in a similar way as $\operatorname{SimReL}(\mathcal{D})$ for FPSs. Lines 14-30 check condition 1 by exploiting the modified algorithm SMF'. For the moment we fix the pair $\left(s_{1}, s_{2}\right) \in R$. For $s_{1} \stackrel{\alpha}{\rightarrow} \mu_{1}$, a boolean variable match $_{\alpha}$ is introduced, which is initialised to false (line 16), and has value true iff $\operatorname{SMF}^{\prime}\left(s_{1}, \alpha, \mu_{1}, s_{2}, \mu_{2}\right)$ returns true on the set $D_{l}^{\left(s_{1}, \alpha, \mu_{1}, s_{2}, \mu_{2}\right)}$ (lines 19-20). In this case, we break the while loop (line 21), and continue to check the next successor distribution of $s_{1}$. If $\operatorname{SMF}^{\prime}\left(s_{1}, \mu_{1}, s_{2}, \mu_{2}, \alpha\right)$ returns false, we remove the first element of $\operatorname{Sim}_{\left(s_{1}, \alpha, \mu_{1}\right)}\left(s_{2}\right)$ (line 22), and take the next candidate of $\mu_{2}$ (line 26) if the set $\operatorname{Sim}_{\left(s_{1}, \alpha, \mu_{1}\right)}\left(s_{2}\right)$ is not empty (line 23). If it is empty, we can not find an $\alpha$-successor distribution related to $\mu_{1}$, so the variable match $_{\alpha}$ remains false. In this case the pair $\left(s_{1}, s_{2}\right)$ does not survive this iteration, and will be dropped out later at line 30. Assume now that the set $\operatorname{Sim}_{\left(s_{1}, \alpha, \mu_{1}\right)}\left(s_{2}\right)$ is not empty. In this case the set $D_{l}^{\left(s_{1}, \alpha, \mu_{1}, s_{2}, \mu_{2}\right)}$ is then reset to $\emptyset$ (line 27 ), and the network, preflow, distance function are initialised (lines 28-29) for the new candidate $\mu_{2}$. Then, we start from the beginning of the while loop, and check if the new candidate $\mu_{2}$ satisfies the condition $\mu_{1} \sqsubseteq_{R} \mu_{2}$. Note that at line 30, the condition is true if and only if match $_{\alpha}$ is true for all $\alpha \in \operatorname{Act}\left(s_{1}\right)$. In this case condition 1 is satisfied and we 


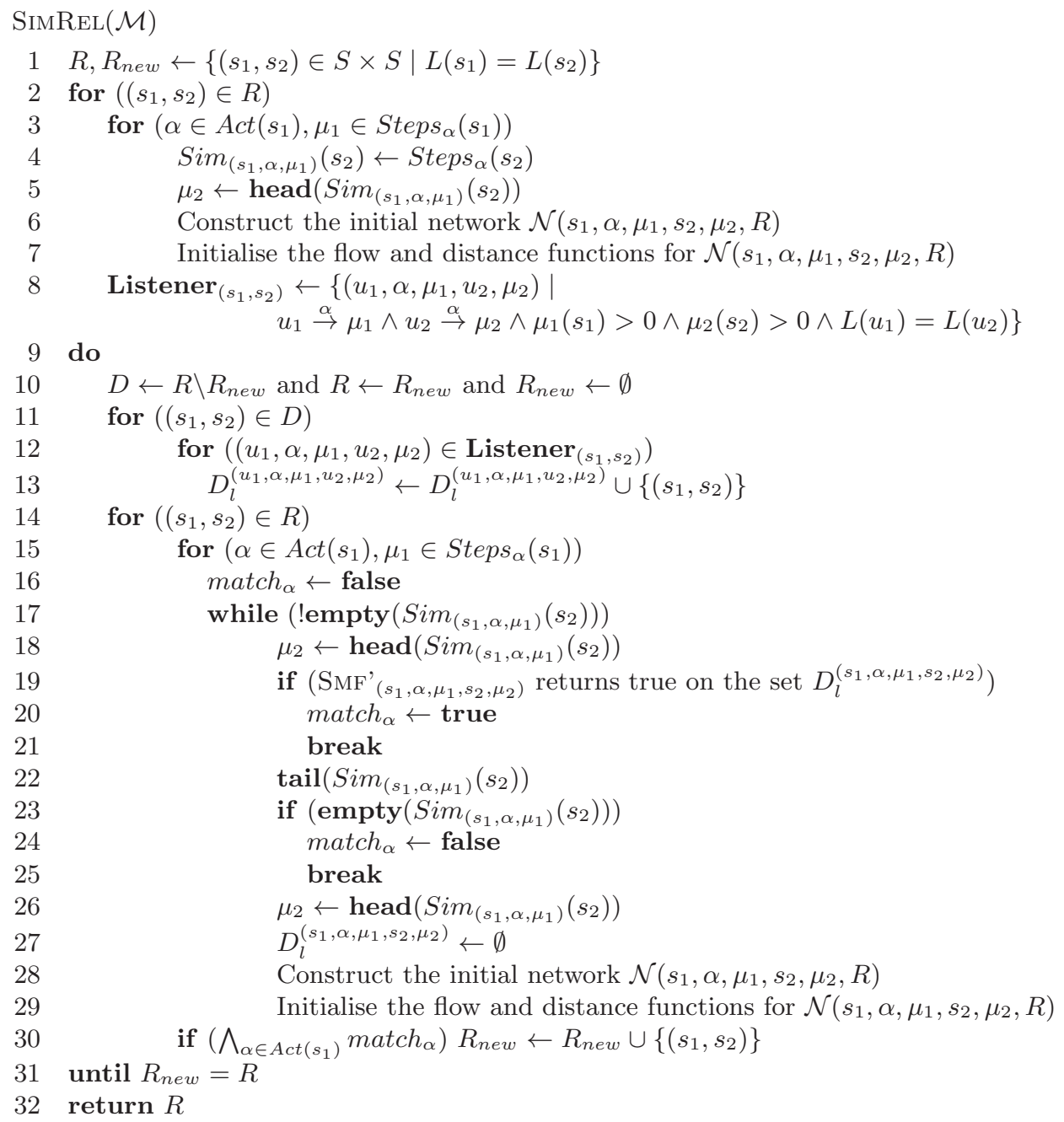

Fig. 2. Efficient algorithm for deciding strong simulation for PAs

insert the pair $\left(s_{1}, s_{2}\right)$ to $R_{\text {new }}$ (line 30$)$. Similar to the algorithm for FPSs, the invariant throughout the loop is that $R$ is coarser than $\precsim \mathcal{M}$. Hence, we obtain the preorder $\precsim \mathcal{M}=R$, once the algorithm terminates (line 32).

Let $n$ denote the number of states, and $m=\sum_{s \in S} \sum_{\alpha \in A c t(s)} \sum_{\mu \in \text { Steps }_{\alpha}(s)}|\mu|$ denote the number of transitions. We give the complexity of the algorithm:

Lemma 5. $\operatorname{SimReL}(\mathcal{M})$ runs in time $\mathcal{O}\left(m^{2} n\right)$ and in space $\mathcal{O}\left(\mathrm{m}^{2}\right)$. If the fanout of $\mathcal{M}$ is bounded by a constant, it has complexity $\mathcal{O}\left(n^{2}\right)$, both in time and space.

Remark 1. For a PA $\mathcal{M}=(S, A c t, \mathbf{P}, L)$, let $m_{b}=\sum_{s \in S} \sum_{\alpha \in A c t(s)}\left|\operatorname{Steps}_{\alpha}(s)\right|$. The algorithm for deciding strong simulation introduced by Baier et. al. has 
time complexity $\mathcal{O}\left(\left(m_{b} n^{6}+m_{b}^{2} n^{3}\right) / \log n\right)$, and space complexity $\mathcal{O}\left(m_{b}^{2}\right)$. Note that $m_{b}$ and $m$ are related by $m_{b} \leq m \leq n m_{b}$. The left equality is established if $|\mu|=1$ for all distributions, and the right equality is established if $|\mu|=n$ for all distributions. Note that for sparse models, we have $m=k m_{b}$ for some $k \in \mathbb{N}$.

Strong Probabilistic Simulations. We now consider finding an algorithm for deciding strong probabilistic simulation. We show that it can be computed by solving LP problems which are decidable in polynomial time $[11,5]$. Recall that strong probabilistic simulation is a relaxation of strong simulation in the sense that it enables combined transitions, which are convex combinations of multiple distributions belonging to equally labelled transitions. Again, the most important part is to check the condition $s_{1} \precsim_{R}^{p} s_{2}$. By Definition 6 , it suffices to check $L\left(s_{1}\right)=L\left(s_{2}\right)$ and the condition:

$$
\forall s_{1} \stackrel{\alpha}{\rightarrow} \mu_{1} . \exists s_{2} \stackrel{\alpha}{\rightarrow} C \mu_{2} \text { with } \mu_{1} \sqsubseteq_{R} \mu_{2}
$$

Since the combined transition involves the quantification of the constants $c_{i}$ ranging over reals, the maximum flow approach for checking $\mu_{1} \sqsubseteq_{R} \mu_{2}$ cannot be applied directly to check $s_{1} \precsim_{R}^{p} s_{2}$. The following lemma shows that condition 2 can be checked by solving LP problems.

Lemma 6. For a given PA, $s_{1} \precsim_{R}^{p} s_{2}$ iff $L\left(s_{1}\right)=L\left(s_{2}\right)$ and for each transition $s_{1} \stackrel{\alpha}{\rightarrow} \mu$, the following LP has a solution:

$$
\begin{aligned}
& \sum_{i=1}^{k} c_{i}=1 \\
& 0 \leq c_{i} \leq 1 \forall i=1, \ldots, k \\
& 0 \leq f_{(s, t)} \leq 1 \quad \forall(s, t) \in R \\
& \mu(s)=\sum_{t \text { with }(s, t) \in R} f_{(s, t)} \quad \forall s \in S \\
& \quad \sum_{s \text { with }(s, t) \in R} f_{(s, t)}=\sum_{i=1}^{k} c_{i} \mu_{i}(t) \quad \forall t \in S
\end{aligned}
$$

where $k=\mid$ Steps $_{\alpha}\left(s_{2}\right) \mid$ and $\operatorname{Steps}_{\alpha}\left(s_{2}\right)=\left\{\mu_{1}, \ldots, \mu_{k}\right\}$.

We introduce a predicate $\operatorname{LP}\left(s_{1}, \alpha, \mu, s_{2}\right)$ which is true iff the above LP problem has a solution. Intuitively, the variables $c_{i}$ correspond to the constants for the combined transition. Constraints 3 and 4 correspond to the requirements of these constants in Definition 5. For every pair $(s, t) \in R$, a variable $f_{(s, t)}$ ranging over [0,1] (Equation 5) is introduced, whose value corresponds to the value of the weight function for $(s, t)$. Equations 6 and 7 establish the weight function conditions of the strong probabilistic simulation. Any solution of the LP problem induces $c_{1}, \ldots, c_{k}$ from which we can construct the desired combined transitions $\mu_{c}=\sum_{i=1}^{k} c_{i} \mu_{i}$ satisfying $\mu \sqsubseteq_{R} \mu_{c}$. Assuming that $L\left(s_{1}\right)=L\left(s_{2}\right)$, then, $s_{1} \precsim_{R}^{p} s_{2}$ iff the conjunction $\bigwedge_{\alpha \in \operatorname{Act}\left(s_{1}\right)} L P\left(s_{1}, \alpha, \mu, s_{2}\right)$ is true. 


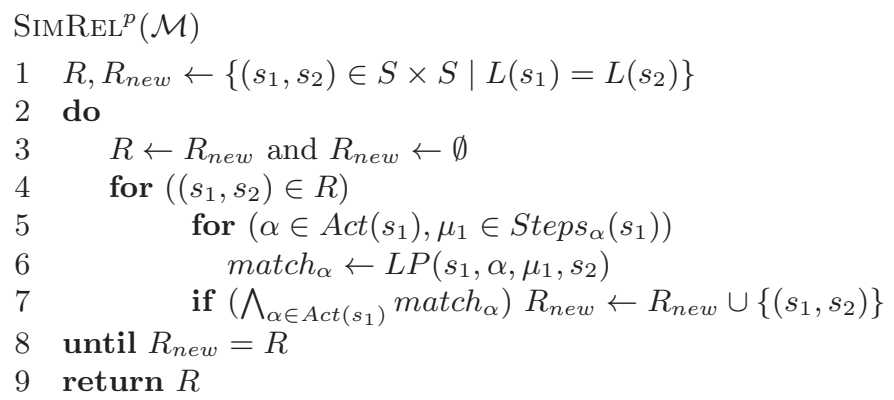

Fig. 3. Algorithm for deciding strong probabilistic simulation for PAs

The algorithm, denoted by $\operatorname{SimReL}^{p}(\mathcal{M})$, is depicted in Fig. 3. It takes the skeleton of algorithm $\operatorname{SimReL}(\mathcal{M})$. The key difference is that we incorporate the predicate $\operatorname{LP}\left(s_{1}, \alpha, \mu, s_{2}\right)$ in line 6 . The correctness of the algorithm $\operatorname{SimReL}^{p}(\mathcal{M})$ is thus similar to the one of $\operatorname{SimReL}(\mathcal{M})$. We discuss the complexity. The number of variables in the LP problem in Lemma 6 is $k+|R|$, and the number of constraints is $1+k+|R|+2|S| \in \mathcal{O}(|R|)$. In every iteration of $\operatorname{SimREL}^{p}(\mathcal{M})$, for $\left(s_{1}, s_{2}\right) \in R$ and $s_{1} \stackrel{\alpha}{\rightarrow} \mu_{1}$, this core is queried one time. The number of iterations is bounded by $\left|R_{\text {init }}\right| \in \mathcal{O}\left(n^{2}\right)$. Therefore, in the worst case, one has to solve $n^{2} \sum_{s \in S} \sum_{\alpha \in \operatorname{Act}(s)} \sum_{\mu \in \text { Steps }_{\alpha}(s)} 1 \leq n^{2} m$ many such LP problems and each of them has maximal $\mathcal{O}\left(n^{2}\right)$ constraints.

Strong Simulation Equivalence. For DTMCs, strong simulation equivalence and strong bisimulation coincide [4, Proposition 3.5]. This is not true for PAs, where strong (probabilistic) simulation equivalence is strictly coarser than strong (probabilistic) bisimulation. Since it is coarser, the induced strong (probabilistic) simulation quotient is potentially again smaller. This is not surprising, as PAs subsume labelled transition systems, in which strong simulation equivalence is strictly coarser than strong bisimulation. The presented decision algorithm can be used to obtain strong simulation equivalence $\precsim \mathcal{M} \cap{ }_{\mathcal{M}}^{-1}$ with complexity $\mathcal{O}\left(m^{2} n\right)$, and to obtain strong probabilistic simulation equivalence $\precsim_{\mathcal{M}}^{p} \cap$ $\left(\precsim_{\mathcal{M}}^{p}\right)^{-1}$ via solving LP problems.

This is directly useful for model checking. We discuss briefly which classes of properties are preserved by strong (probabilistic) simulation equivalence. We assume acquaintance with the logic PCTL, particularly the safe and live fragments of PCTL. For more detail, we refer to [4]. Strong (probabilistic) simulation is known to preserve all safe fragments of PCTL [12], which can be lifted to the quotient automaton:

Lemma 7. For PAs, the strong (probabilistic) simulation quotient automaton preserves both safe and live fragments of PCTL formulas.

Recall that for MDPs, strong simulation and strong probabilistic simulation trivially coincide. More surprisingly, strong simulation equivalence and strong bisimulation also coincide on MDPs [1, Theorem 3.4.15]. Therefore, as a byproduct of 
algorithm $\operatorname{SimReL}(\mathcal{M})$, we obtain a decision algorithm for strong bisimulation of MDPs: strong bisimulation for an MDP $\mathcal{M}$ can be obtained by $\precsim_{\mathcal{M}} \cap \precsim_{\mathcal{M}}^{-1}$, with complexity $\mathcal{O}\left(m^{2} n\right)$.

\section{Algorithms for Continuous-Time Probabilistic Automata}

In this section we introduce a generalisation of PAs where the transitions are described by rates instead of probabilities. Then, we extend the decision algorithms to continuous-time models. For that purpose we let $r: S \rightarrow \mathbb{R}_{\geq 0}$ denote the rate function, and let Rate $(S)$ denote the set of all rate functions.

Definition 7. A continuous-time PA (CPA) is a tuple $(S, A c t, \mathbf{R}, L)$ where $S$, Act, $L$ as defined for $P A s$, and $\mathbf{R} \subseteq S \times A c t \times \operatorname{Rate}(S)$ a finite set, called the rate matrix.

We write $s \stackrel{\alpha}{\rightarrow} r$ if $(s, \alpha, r) \in \mathbf{R}$. The model continuous-time Markov decision processes (CTMDPs) [10,3] can be considered as special CPAs where for $s \in S$ and $\alpha \in A c t$, there exists at most one rate function $r \in \operatorname{Rate}(S)$ such that $s \stackrel{\alpha}{\rightarrow} r$. The model CTMDPs considered in paper [13] essentially agrees with our CPAs. Note that CPAs generalise PAs in a similar way as CTMDPs generalise MDPs.

Strong Simulations. For a rate function $r$ with $r(S)>0$, we let $\mu(r) \in \operatorname{Dist}(S)$ denote the induced distribution defined by: $\mu(r)(s)$ equals $r(s) / r(S)$ if $r(S)>0$ and 0 otherwise. Now we introduce the notion of strong simulation for CPAs, which can considered as an extension of the definition for CTMCs [4]:

Definition 8. Let $\mathcal{M}=(S, A c t, \mathbf{R}, L)$ be a $C P A . R \subseteq S \times S$ is a strong simulation on $\mathcal{M}$ iff for all $s_{1}, s_{2}$ with $s_{1} R s_{2}: L\left(s_{1}\right)=L\left(s_{2}\right)$ and if $s_{1} \stackrel{\alpha}{\rightarrow} r_{1}$ then there exists a transition $s_{2} \stackrel{\alpha}{\rightarrow} r_{2}$ with $\mu\left(r_{1}\right) \bigsqcup_{R} \mu\left(r_{2}\right)$ and $r_{1}(S) \leq r_{2}(S)$. We write $s_{1} \precsim \mathcal{M} s_{2}$ iff there exists a strong simulation $R$ on $\mathcal{M}$ such that $s_{1} R s_{2}$.

The additional rate condition $r_{1}(S) \leq r_{2}(S)$ indicates that $r_{2}$ is faster than $r_{1}$. The decision algorithm for strong simulation can be adapted from algorithm SimReL in Fig. 2 easily: We replace every occurrence of $\mu_{1}$ and $\mu_{2}$ by $r_{1}$ and $r_{2}$, respectively. Other notations are extended with respect to rate functions in an obvious way. To guarantee the additional rate condition, we rule out transitions that violate it by replacing line 4 by: $\operatorname{Sim}_{\left(s_{1}, \alpha, r_{1}\right)}\left(s_{2}\right) \leftarrow\left\{r_{2} \in \operatorname{Steps}_{\alpha}\left(s_{2}\right) \mid\right.$ $\left.r_{1}(S) \leq r_{2}(S)\right\}$. Obviously, the so obtained algorithm for CPAs has the same complexity $\mathcal{O}\left(m^{2} n\right)$ as for PAs.

Strong Probabilistic Simulations. We extend the notion of strong probabilistic simulation to CPAs. Firstly, we introduce combined transitions for CPAs:

Definition 9. Let $\mathcal{M}=(S, A c t, \mathbf{R}, L)$ be a CPA. Assume that $\left\{r_{1}, \ldots, r_{k}\right\} \subseteq$ Steps $_{\alpha}(s)$ where $r_{i}(S)=r_{j}(S)$ for $i, j \in\{1, \ldots, n\}$. The tuple $(s, \alpha, r)$ is a combined transition, denoted by $s \stackrel{\alpha}{\rightarrow}_{C} r$, iff there exist constants $c_{1}, \ldots, c_{k} \in$ $[0,1]$ with $\sum_{i=1}^{k} c_{i}=1$ such that $r(s)=\sum_{i=1}^{k} c_{i} r_{i}(s)$ for all $s \in S$. 
In the above definition, unlike for the PA case, only $\alpha$-successor rate functions with same exit rate can be combined together. This restriction makes the combined transition also exponential distributed. Without this restriction, the combined transition is not exponential distributed any more, precisely, it is hyper-exponential distributed. To see this we consider $S=\left\{s_{1}, s_{2}, s_{3}\right\}$, and $s_{1} \stackrel{\alpha}{\rightarrow} r_{1}$ and $s_{1} \stackrel{\alpha}{\rightarrow} r_{2}$. The rate function $r_{1}$ is defined by $r_{1}\left(s_{2}\right)=2, r_{1}\left(s_{3}\right)=8$, and $r_{2}$ is defined by $r_{2}\left(s_{2}\right)=12, r_{2}\left(s_{3}\right)=6$. Taking $r_{1}$ and $r_{2}$ with equal probability 0.5 , we would get the combined transition $r=0.5 r_{1}+0.5 r_{2}$ satisfying: $r\left(s_{2}\right)=7$ and $r\left(s_{3}\right)=7$. If $r$ is exponential distributed, we would expect that the probability of reaching state $s_{2}$ within time $t$ should be $\frac{7}{14} *\left(1-\exp ^{-14 t}\right)$. However, the combined transition is hyper-exponential distributed: the probability of reaching state $s_{2}$ within time $t$ under $r$ is given by:

$$
0.5 * \frac{2}{10} *\left(1-\exp ^{-10 t}\right)+0.5 * \frac{12}{18} *\left(1-\exp ^{-18 t}\right)
$$

Similarly, the probability of reaching state $s_{3}$ within time $t$ is given by: $0.5 * \frac{8}{10} *$ $\left(1-\exp ^{-10 t}\right)+0.5 * \frac{6}{18} *\left(1-\exp ^{-18 t}\right)$.

Similar to PAs, strong probabilistic simulation is insensitive to combined transitions, is thus a relaxation of strong simulation:

Definition 10. Let $\mathcal{M}=(S$, Act, $\mathbf{R}, L)$ be a CPA. $R \subseteq S \times S$ is a strong probabilistic simulation on $\mathcal{M}$ iff for all $s_{1}, s_{2}$ with $s_{1} R s_{2}: L\left(s_{1}\right)=L\left(s_{2}\right)$ and if $s_{1} \stackrel{\alpha}{\rightarrow} r_{1}$ then there exists a combined transition $s_{2} \stackrel{\alpha}{\rightarrow}_{C} r_{2}$ with $\mu\left(r_{1}\right) \sqsubseteq_{R} \mu\left(r_{2}\right)$ and $r_{1}(S) \leq r_{2}(S)$. We write $s_{1} \precsim_{\mathcal{M}}^{p} s_{2}$ iff there exists a strong simulation $R$ on $\mathcal{M}$ such that $s_{1} R s_{2}$.

The notation of simulation up to $R$ for strong probabilistic simulation can be defined in a similar way as for PAs. To check the condition $s_{1} \precsim_{R}^{p} s_{2}$ for the CPA $\mathcal{M}$ we resort to a reduction to LP problems:

Lemma 8. For a given CPA, $s_{1} \precsim_{R}^{p} s_{2}$ iff $L\left(s_{1}\right)=L\left(s_{2}\right)$ and for each transition $s_{1} \stackrel{\alpha}{\rightarrow} r$ there exists $\left\{r_{1}, \ldots, r_{k}\right\} \subseteq \operatorname{Steps}_{\alpha}\left(s_{2}\right)$ with $r_{i}(S)=r_{j}(S)$ and $r_{i}(S) \geq$ $r(S)$ for $i, j \in\{1, \ldots, k\}$ such that the following LP has a solution, which consists of constraints 3, 4, 5 of Lemma 6, and additionally:

$$
\begin{aligned}
& r(s)=r(S) \sum_{t \text { with }(s, t) \in R} f_{(s, t)} \quad \forall s \in S \\
& r_{1}(S) \sum_{s \text { with }(s, t) \in R} f_{(s, t)}=\sum_{i=1}^{k} c_{i} r_{i}(t) \quad \forall t \in S \\
& r(S) \leq r_{1}(S)
\end{aligned}
$$

Similar to Lemma 6 , for every $E \in\left\{r^{*}(S) \mid s_{2} \stackrel{\alpha}{\rightarrow} r^{*}\right\}$ with $E>r_{1}(S)$ we introduce the predicate $L P^{\prime}\left(s_{1}, \alpha, r, s_{2}, E\right)$ which is true iff the above LP problem has a solution. In comparison to the LP problem in Lemma 6, Equation 10 
establishes the rate condition. Recall that $\mu(r)(s)=r(s) / r(S)$. Equation 8 corresponds to Equation 6 where $r(S)$ is multiplied on both sides. Now we consider Equation 9. Let $r_{c}(t)=\sum_{i=1}^{k} c_{i} r_{i}(t)$ be the combined transition that simulates $r$. Note $\mu\left(r_{c}\right)(t)=\sum_{i=1}^{k} c_{i} r_{i}(t) / \sum_{i=1}^{k} c_{i} r_{i}(S)$. Since we have $r_{i}(S)=r_{j}(S)$ for all $i, j \in\{1, \ldots, k\}$, the denominator is simplified to $r_{1}(S)$. Hence, Equation 9 corresponds to Equation 7 where $r_{1}(S)$ is multiplied on both side. Note that the denominator could not be simplified without the restriction in the combined transition, i. e., only rate function with the same exit rate can be combined. Equation 9 would not be a linear constraint any more for this case. Obviously, the LP problem has a solution iff there is such a combined transition $r_{c}$ satisfying the conditions $\mu(r) \sqsubseteq_{R} \mu\left(r_{c}\right)$ and $r(S) \leq r_{c}(S)$. Assuming that $L\left(s_{1}\right)=L\left(s_{2}\right)$, then, $s_{1} \precsim_{R}^{p} s_{2}$ iff the conjunction $\bigwedge_{\alpha \in \operatorname{Act}\left(s_{1}\right)} L P^{\prime}\left(s_{1}, \alpha, r, s_{2}, E\right)$ is true for an $E \in\left\{r^{*}(S) \mid s_{2} \stackrel{\alpha}{\rightarrow} r^{*} \wedge r^{*}(S) \geq r_{1}(S)\right\}$. The decision algorithm can be obtained by replacing the predicate $L P\left(s_{1}, \alpha, \mu, s_{2}\right)$ by $L P^{\prime}\left(s_{1}, \alpha, r, s_{2}, E\right)$ of algorithm $\operatorname{SimReL}^{p}(\mathcal{M})$ in Fig. 3 where $E$ ranges over $\left\{r^{*}(S) \mid s_{2} \stackrel{\alpha}{\rightarrow} r^{*} \wedge r^{*}(S) \geq r_{1}(S)\right\}$. As complexity we have to solve $n^{2} m$ LP problems and each of them has maximal $\mathcal{O}\left(n^{2}\right)$ constraints.

Strong Simulation Equivalence. Similar to PAs, as a byproduct we obtain a decision algorithm for strong (probabilistic) simulation equivalence for CPAs. We discuss also which classes of properties are preserved by strong (probabilistic) simulation equivalence. We assume acquaintance with the logic CSL, particularly the safe and live fragments of CSL. For more detail, we refer to [4]. Now we give the continuous-time counterpart of Lemma 7 for CPAs:

Lemma 9. For CPAs, the strong (probabilistic) simulation preserves all safe fragments of CSL formulas. Moreover, the strong (probabilistic) simulation quotient automaton preserves both safe and live fragments of CSL formulas.

For CTMDPs, strong simulation and strong probabilistic simulation also trivially coincide, as for MDPs. Another similar result is that the strong simulation quotient and the strong bisimulation for CTMDPs also coincide.

\section{Conclusion}

We presented algorithms for computing simulation preorders for PAs. We achieved an algorithm with complexity $\mathcal{O}\left(m^{2} n\right)$ for strong simulation. For strong probabilistic simulation, we have shown that the preorder can be determined by solving LP problems. We extended the algorithms to CPAs with same complexities for both strong simulation and strong probabilistic simulation. As further work, we would like to extend our results to weak (probabilistic) simulations for PAs and CPAs.

Acknowledgement. We thank Martin Neuhäußer for pointing out an error in the definition of combined transitions for CPAs in an earlier version of this paper. 


\section{References}

1. C. Baier. On Algorithmic Verification Methods for Probabilistic Systems, 1998. Habilitations-schrift zur Erlangung der venia legendi der Fakultät für Mathematik and Informatik, Universität Mannheim.

2. C. Baier, B. Engelen, and M. E. Majster-Cederbaum. Deciding bisimilarity and similarity for probabilistic processes. J. Comput. Syst. Sci., 60(1):187-231, 2000.

3. C. Baier, H. Hermanns, J.-P. Katoen, and B. R. Haverkort. Efficient computation of time-bounded reachability probabilities in uniform continuous-time markov decision processes. Theor. Comput. Sci., 345(1):2-26, 2005.

4. C. Baier, J.-P. Katoen, H. Hermanns, and V. Wolf. Comparative branching-time semantics for markov chains. Inf. Comput, 200(2):149-214, 2005.

5. S. Boyd and L. Vandenberghe. Convex Optimization. Cambridge University Press, 2004.

6. S. Cattani and R. Segala. Decision algorithms for probabilistic bisimulation. In CONCUR, pages 371-385, 2002.

7. G. Gallo, M.D. Grigoriadis, and R.E. Tarjan. A fast parametric maximum flow algorithm and applications. SIAM J. Comput., 18(1):30-55, 1989.

8. B. Jonsson and K.G. Larsen. Specification and refinement of probabilistic processes. In $L I C S$, pages 266-277, 1991.

9. K. G. Larsen and A. Skou. Bisimulation through probabilistic testing. Inf. Comput., 94(1):1-28, 1991.

10. M. L. Puterman. Markov Decision Processes: Discrete Stochastic Dynamic Programming. John Wiley \& Sons, 1994.

11. A. Schrijver. Theory of Linear and Integer Programming. Wiley, 1986.

12. R. Segala and N. A. Lynch. Probabilistic simulations for probabilistic processes. Nord. J. Comput., 2(2):250-273, 1995.

13. N. Wolovick and S. Johr. A characterization of meaningful schedulers for continuous-time markov decision processes. In FORMATS, pages 352-367, 2006.

14. L. Zhang, H. Hermanns, F. Eisenbrand, and D.N. Jansen. Flow faster: Efficient decision algorithms for probabilistic simulations. In TACAS, pages 155-169, 2007. 